\title{
Magnetic Properties of Yttrium-Substituted NiZn Ferrites
}

\author{
V. JANČÁRIK*, E. UšÁK, M. ŠOKA, M. UŠÁKOVÁ \\ Institute Electrical Engineering, Faculty of Electrical Engineering and Information Technology, Slovak University of \\ Technology, Ilkovičova 3, 81219 Bratislava, Slovakia

\begin{abstract}
Polycrystalline NiZn ferrite doped with a small amount of $\mathrm{Y}^{3+}$ ions and having the composition of $\mathrm{Ni}_{0.42} \mathrm{Zn}_{0.58} \mathrm{Y}_{x} \mathrm{Fe}_{2-x} \mathrm{O}_{4}$, where $x=0.00,0.01,0.02,0.04,0.06,0.08$ and 0.10 , was analyzed. Specimens were prepared by the ceramic method. Thermo-magnetic analysis and evaluation of hysteresis loops parameters were chosen as main examination methods in this study. Various parameters, such as Curie temperature $T_{C}$, coercive field $H_{c}$, remanent magnetic flux density $B_{r}$, hysteresis loop area and amplitude/initial permeability were studied at low frequencies.
\end{abstract}

DOI: 10.12693/APhysPolA.126.90

PACS: 75.50.Gg, 75.30.Cr, 75.60.-d, 74.62.Dh

\section{Introduction}

NiZn ferrites still belong to a group of interesting magnetic materials for high-frequency applications thanks to their relatively high initial permeability, sufficient saturation magnetization and low power losses. Substitutions are investigated to improve parameters important from point of view of applications of these materials. The substitution changes the chemical composition and the structure of ferrite and then consequently it yields changes of magnetic parameters. Substitutions by rare-earth ions are made to improve properties of these ferrites. They are affected by chemical composition, i.e. type and amount of the substituent, as well as sintering conditions, grain size, impurities and the preparation procedure.

Several substitutions by rare-earth ions were tested [1-2]. The addition of a small amount of these ions influences the magnetic parameters of the ferrite due to changes of magneto-crystalline anisotropy through rareearth-Fe interaction [3] mainly.

\section{Specimen preparation and experiment}

Samples of $\mathrm{Ni}_{0.42} \mathrm{Zn}_{0.58} \mathrm{Y}_{x} \mathrm{Fe}_{2-x} \mathrm{O}_{4}$ ferrite compounds $(x=0.00,0.01,0.02,0.04,0.06,0.08$ and 0.10$)$, annealed at $1200{ }^{\circ} \mathrm{C}$, were prepared by ceramic procedure. The mixture of initial raw materials - analytical grade oxides of relevant metals, was homogenized by wet-milling, filtered and dried, next calcined at $950{ }^{\circ} \mathrm{C}$ for $1 \mathrm{~h}$. Afterwards, the product of calcination was thermally treated at $1200{ }^{\circ} \mathrm{C}$ for $6 \mathrm{~h}$. A part of prepared powder samples (approx. $50 \mathrm{mg}$ ) was used for the measurement of temperature dependence of magnetic susceptibility $\chi(T)$. Ring-shaped (toroidal) samples with the outer diameter of $12 \mathrm{~mm}$ and the inner diameter of $6 \mathrm{~mm}$ were employed for measurement of low-frequency hysteresis loops. Such procedure was carried out for each value of yttrium content $x$. The measured temperature dependencies of magnetic susceptibility $\chi(T)$ and low-frequency hysteresis

*corresponding author; e-mail: vladimir.jancarik@stuba.sk loops were chosen for the evaluation of magnetic properties. Temperature dependence of magnetic susceptibility $\chi(T)$ was measured by means of bridge method at frequency $f=760 \mathrm{~Hz} . \quad \chi(T)$ dependence is very sensitive to chemical and structural composition of ferrites. Even if it does not give detailed structural information, such as e.g. X-ray diffraction, it is relatively cheap. We use it as a handy method for bringing quick information about the material [4]. Moreover, Curie temperature $T_{C}$ determined from $\chi(T)$ dependence, as well as $\chi(T)$ dependence itself, describing thermal stability of the ferrite, is important from its application point of view. $T_{C}$, coresponding to the point of inflexion of the $\chi(T)$ dependence, is found by a numerical procedure. The measured points of the $\chi(T)$ curve are fitted by the natural cubic splines around the expected $T_{C}$ and the temperature at which $\partial^{2} \chi(T) / \partial T^{2}=0$ is found. Low-frequency hysteresis loops were recorded on ring-shaped samples using automated digital hysteresisgraph [5]. The power losses, the remanent magnetic flux density $B_{r}$ and the coercive field $H_{c}$ were determined from the hysteresis loops as well. From the families of hysteresis loops, also the amplitude permeability $\mu_{a}$ as a function of exciting field amplitude was evaluated. The initial permeability $\mu_{i}$ was found as an extrapolation of these dependencies to zero fields.

\section{Results and discussion}

The temperature dependences of magnetic susceptibility $\chi(T)$ of prepared $\mathrm{Ni}_{0.42} \mathrm{Zn}_{0.58} \mathrm{Y}_{x} \mathrm{Fe}_{2-x} \mathrm{O}_{4}$ ferrites are shown in Fig. 1. Above the $T_{C}$, the $\chi(T)$ curves shortly fall from relatively high values to near zero, which indicates the presence of single-phase system, consisting of spinel-type ferrite. In addition, the absence of the Hopkinson's peak excludes the occurrence of small singledomain particles in super-paramagnetic state. The Curie temperature increases almost linearly with the increasing $\mathrm{Y}^{3+}$ ions content. Additionally a strong drop of initial permeability appears at room temperature even for a small amount of paramagnetic $\mathrm{Y}^{3+}$ ions (see Fig. 2). This behavior is due to the replacement of $\mathrm{Y}^{3+}$ at the expense of $\mathrm{Fe}^{3+}$ ions, and as the result, of the modification 


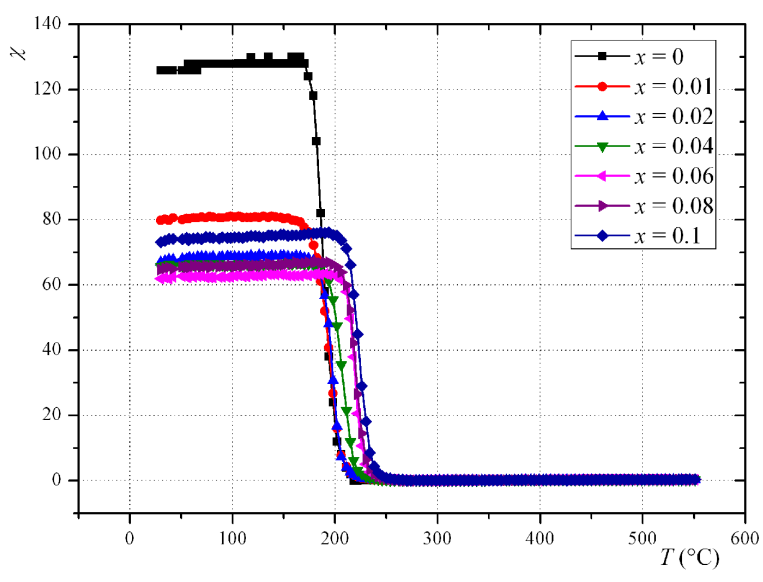

Fig. 1. Temperature dependences of magnetic susceptibility of $\mathrm{Ni}_{0.42} \mathrm{Zn}_{0.58} \mathrm{Y}_{x} \mathrm{Fe}_{2-x} \mathrm{O}_{4}$.

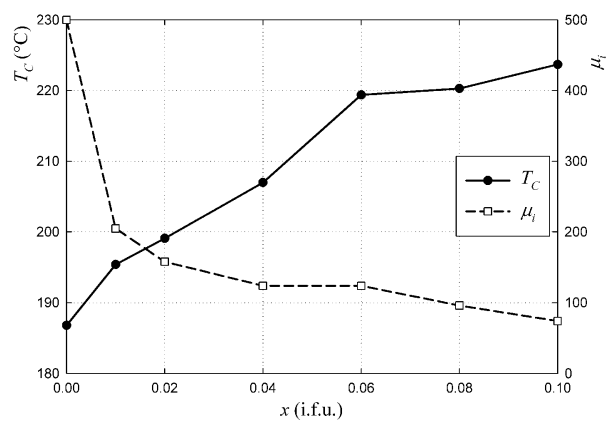

Fig. 2. Dependences of the Curie temperature and the initial permeability on $x$ ions content of $\mathrm{Ni}_{0.42} \mathrm{Zn}_{0.58} \mathrm{Y}_{x} \mathrm{Fe}_{2-x} \mathrm{O}_{4}$.

of A-B exchange interaction strength and the decrease of the overall magnetic moment in accordance with cation distribution formula, where round parentheses ( ) represent $\mathrm{A}$ sites and the square parentheses [] represent B sites of spinel structure:

$$
\left(\mathrm{Zn}_{0.58}^{2+} \mathrm{Fe}_{0.42}^{3+}\right)\left[\mathrm{Ni}_{0.42}^{2+} \mathrm{Y}_{x}^{3+} \mathrm{Fe}_{1.58-x}^{3+}\right] \mathrm{O}_{4}^{2-}
$$

The hysteresis loops of $\mathrm{Ni}_{0.42} \mathrm{Zn}_{0.58} \mathrm{Y}_{x} \mathrm{Fe}_{2-x} \mathrm{O}_{4}$ measured

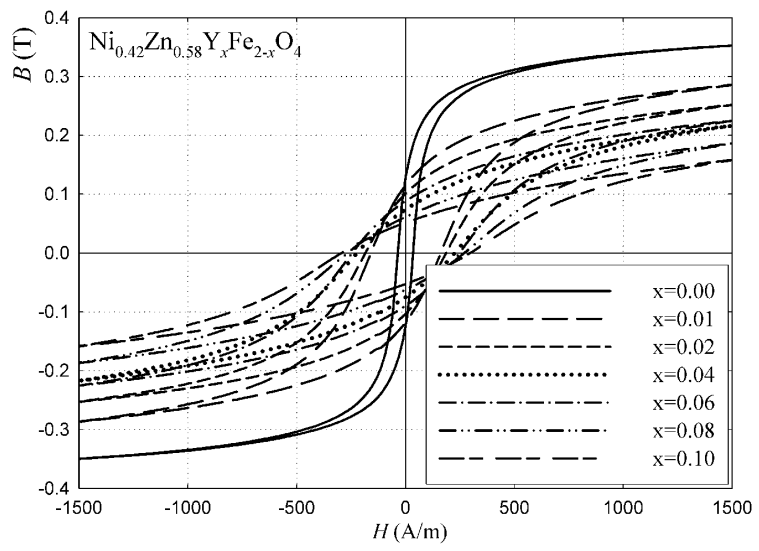

Fig. 3. Hysteresis loops of $\mathrm{Ni}_{0.42} \mathrm{Zn}_{0.58} \mathrm{Y}_{x} \mathrm{Fe}_{2-x} \mathrm{O}_{4}$ ferrites.

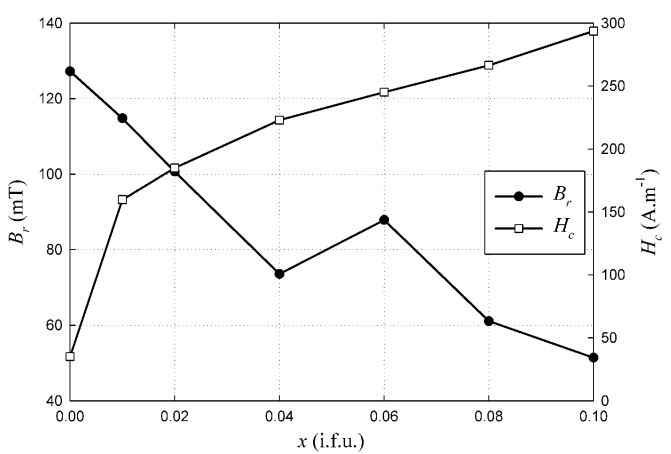

Fig. 4. Remanent flux density and coercivity of $\mathrm{Ni}_{0.42} \mathrm{Zn}_{0.58} \mathrm{Y}_{x} \mathrm{Fe}_{2-x} \mathrm{O}_{4}$ for various $\mathrm{Y}$ substitutions $x$.

at frequency $f=50 \mathrm{~Hz}$, are shown in Fig. 3. The magnetization characteristics depend significantly on relatively small amount of Y substitution.

One can observe large differences in coercivity, as well as the remanence (see Fig. 4). The loops become wider (the coercivity increases), meanwhile the remanent flux density decreases. All these changes can apparently be associated with reordering of ions in both sublattices of the spinel structure due to large difference between ionic radii of $\mathrm{Y}$ and $\mathrm{Fe}$.

\section{Conclusions}

Magnetic properties of Y-doped NiZn ferrites were analyzed. Because of the large radii, the $\mathrm{Y}$ ions incorporated in the ferrite cause slight deformation of the crystalline array as well as reordering of Fe ions in both sublattices. This leads to the increase of Curie temperature and coercivity, whereas the initial susceptibility and the remanent flux density decrease.

\section{Acknowledgments}

This work was supported by the Slovak Research and Development Agency under the contract No. APVV0062-11 and by Scientific Grant Agency of the Ministry of Education of the Slovak Republic and the Slovak Academy of Sciences (VEGA) (projects No. VG$1 / 1163 / 12$ and VG-1/1325/12).

\section{References}

[1] S.E. Jacobo, S. Duhalde, H.R. Bertorello, J. Magn. Magn. Mater. 227-276, 2253 (2004).

[2] J. Sun, J. Li, G. Sun, J. Magn. Magn. Mater 250, 20 (2002).

[3] Y.C. Yang, L.S. Kong, S.H. Sun, D.M. Gu, B.P. Cheng, J. Appl. Phys. 63, 3702 (1988).

[4] V. Jančárik, E. Ušák, Journal of Electrical Engineering 50, 63 (1999).

[5] E. Ušák, Advances in Electrical and Electronic Engineering 11, 116 (2013). 